\title{
Is there a room for immune checkpoint inhibitors in early stage non-small cell lung cancer?
}

\author{
Elisa Gobbini' ${ }^{1}$, Matteo Giaj Levra ${ }^{2}$ \\ ${ }^{1}$ Department of Oncology, University of Turin, AOU San Luigi Gonzaga, Orbassano, Italy; ${ }^{2}$ Thoracic Oncology Unit, CHU Grenoble, La Tronche, \\ France \\ Contributions: (I) Conception and design: All authors; (II) Administrative support: None; (III) Provision of study materials or patients: All authors; \\ (IV) Collection and assembly of data: All authors; (V) Data analysis and interpretation: All authors; (VI) Manuscript writing: All authors; (VII) Final \\ approval of manuscript: All authors. \\ Correspondence to: Dr. Elisa Gobbini. Department of Oncology, AOU San Luigi Gonzaga, Regione Gonzole 10, 10043 Orbassano (TO), Italy. \\ Email: elisa.gobbini@hotmail.it.
}

\begin{abstract}
Early non-small cell lung cancer (NSCLC) represents $16 \%$ of all new NSCLC at diagnosis with a 5 -year survival rate of about $60 \%$. Surgical intervention and adjuvant platinum-based chemotherapy represent the cornerstone treatments, but no significant advances have been achieved since several decades in term of relapse rate reduction or survival improvement. Immunotherapy represents an appealing strategy considering the acceptable toxicity profile but, despite the awesome changing recently introduced in the locally advanced and metastatic setting, its role in early NSCLC is not clear yet. In the past few years, two strategies have been investigated to improve the early NSCLC outcomes eliciting the anti-tumour immune response: tumour vaccines and adoptive cellular therapies. However, none of them provided convincing results. Preclinical and clinical data supported the prognostic role of immune checkpoints in resected NSCLC even if they did not show a clear predictive value for adjuvant treatment. However, some preliminary data about safety and efficacy of neo-adjuvant immune checkpoint inhibitors encourage further investigation of their potential role as monotherapy or as part of a multimodal strategy. Then, even if no significant progress has been done in early NSCLC treatment until today, checkpoint inhibitors can open the door to a new strategy in this setting.
\end{abstract}

Keywords: Lung neoplasms; immunotherapy; adoptive transfer; vaccine; adjuvant therapy

Submitted Dec 31, 2017. Accepted for publication Jan 09, 2018.

doi: $10.21037 /$ jtd.2018.01.81

View this article at: http://dx.doi.org/10.21037/jtd.2018.01.81

\section{Introduction}

Non-small cell lung cancer (NSCLC) is the leading cause of cancer mortality representing about the $25 \%$ of all cancer death. Unfortunately, most of patients present a locally advanced or metastatic disease at diagnosis and only $16 \%$ of all NSCLC are confined to the lung. The 5 -year survival rate of this small group of patients is about $60 \%(1,2)$. Surgical resection represents the cornerstone treatment for early NSCLC and the platinum-based adjuvant chemotherapy confers an absolute 5 -year survival improvement of $4-5 \%(3,4)$. Therefore, proper recovery from surgery and the absence of major comorbidities are the essential features to consider in patients' selection to adjuvant treatment. For patients with severe comorbidities or other reasons of inoperability, stereotactic radiotherapy (SABR) is an alternative option, with a local control rate of $90 \%$ at 5 years $(5,6)$. In any case, patients present during the first 4 years after surgery a high risk of relapse ranging from $6 \%$ to $10 \%$ per person per year, justifying a strict surveillance (7).

No significant therapeutic innovations have been introduced in early NSCLC management since several decades, and no advances in relapse reduction and survival 
improvement have been achieved. Immunotherapy represents an appealing strategy considering the acceptable toxicity profile but, despite the awesome changing recently introduced in metastatic setting thanks to the approval of pembrolizumab and nivolumab (8-10), and in locally advanced disease with durvalumab maintenance after concomitant radio-chemotherapy (11), the role of immuneoncology in early NSCLC management is not clear yet. This paper aims to review the rational to investigate the role of immunotherapy in early NSCLC and the results of more significant studies performed in this setting.

\section{Rational for immunotherapy in early NSCLC}

\section{Prognostic role of immune cells infiltrating resected NSCLC specimens}

Tumours are not only composed by malignant cells but also by stromal, endothelial and immune system cells that interact each other in complex ways. Many studies have investigated the correlation between the immune cells infiltrate in the primary tumour and patients' outcome after surgical resection in early stage NSCLC.

In a large cohort of resected specimens, the presence of $\mathrm{T}$ cells in the tumour infiltrate was associated with favourable prognosis, while non-specific immune cells infiltrate did not (12). A favourable effect on prognosis was seen in resected NSCLC infiltrated by CD8+ cytotoxic T cells, and a high density of both CD4+ and CD8+ lymphocytes in the stromal (but not in the epithelial) compartment was associated to a better disease survival in stage I to IIIA (13-15).

Tumour associated macrophages (TAMs) represent a major component of the immune cells infiltrate with a central role in the cross-talking between cancer and immune system (16). M2 macrophages are characterized by an immunosuppressive phenotype and they can facilitate tumour progression, while M1 macrophages promote the anti-tumour immune response exerting a cytotoxic effect on cancer cells (17). Many studies did not separately consider these two cells subsets in prognostic evaluations, achieving contradictory results: some groups reported that TAMs could be associated with favourable prognosis in early stage NSCLC $(14,18)$, while in the largest reported trial this association was not confirmed (19). However, studies evaluating only M1 macrophages reported a better prognosis in early stage NSCLC $(20,21)$.

Finally, the count of dendritic cells (DCs), the most efficient antigen presenting cells (APCs) for inducing an adaptive immune response to cancer, seemed to be correlated with prognosis too. According to two different studies, higher DCs counts in tumour epithelial and stromal compartments of resected NSCLC were associated with absence of nodal involvement and significantly better disease specific survival (DSS). Stage I patients presented more frequently a high DCs count being considered as an independent prognostic factor $(19,22)$.

Moving from a specific cells subset to the general immune tissue organization, some experiences investigated the prognostic role of organized lymphoid structures in resected early NSCLC as a marker of functional anti-tumour adaptive response. In a series of 91 resected early stage NSCLC the relationship between the presence of germinal centres (GCs) in the organized lymphoid structures and survival was evaluated. One third of specimens analysed presented GCs at tumour margins or in its centre. The presence of intratumoral but not marginal GCs was associated with earlier stage and in stage I the presence of intratumoral GCs was associated with better survival compared to no GCs (23). Subsequently, a French series of 74 resected early stage NSCLC was evaluated for the presence of tertiary lymphoid structures, defined as tumour-induced bronchus associated lymphoid tissues (Ti-BALT). Because the TiBALT counting was difficult to assess, authors used mature DCs that homed exclusively in Ti-BALT as a specific marker of these structures. The density of mature DCs was found as a significant predictor of overall, disease specific and diseasefree survival (DFS) (24).

\section{Prognostic role of immune checkpoints expression in resected NSCLC specimens}

Tumours can regulate their immune microenvironment with several mechanisms like the modulation of immune checkpoints or the attraction of immune-suppressor cells as regulatory T cells (Treg), myeloid derived suppressor cells (MDSC) and TAMs (3,25-28). Of special interest, is the ability of tumour cells to up-regulate the surface expression of certain immune checkpoint molecules, promoting immune system tolerance and leading to the immune surveillance escape. The more investigated immune checkpoint receptors are the cytotoxic T-lymphocytesassociated protein-4 (CTLA4) and the programmed cell death protein-1 (PD-1) with its ligands PD-L1 and PD-L2 $(29,30)$.

CTLA4 receptors are normally expressed on activated $\mathrm{T}$ cells as the result of the combination of the early $\mathrm{T}$ cell 
activation and the interleukin 2 stimulation; they are able to bind with high affinity the B7 molecule on the APCs, breaking the $\mathrm{B} 7$ positive costimulatory signal early in the $\mathrm{T}$ cells activation process occurring in lymph nodes (31-33).

In contrast to CTLA4, PD-1 is activated during the effector stages on activated $\mathrm{T}$ cell and the interaction with its ligands occurs in peripheral tissues. Activated $\mathrm{T}$ cells limit uncontrolled cytotoxic $\mathrm{T}$ cell attacks by producing interferon gamma, which induces PD-L1 expression on the APCs but also on tumour cells surface (34). The abnormal expression on the cell surface of checkpoint receptors and their ligands induce $T$ cell anergy, affording tumour protection against host immune system (35).

The prognostic implication of CTLA4 and PD-L1 expression in early NSCLC is debated. It has been described that the high expression of CTLA4 predicts a worst overall survival (OS) in a cohort of stage I to III NSCLC but, on the contrary, others authors founded a reduced death rate in a similar population overexpressing CTLA4 $(29,30)$.

PD-L1 expression was evaluated in two large cohorts of NSCLC including stage I to IV disease, observing that high expression of $\mathrm{PD}-\mathrm{L} 1$ protein or mRNA was associated with a better outcome (36). Other authors reported similar results founding that tumour PD-L1 expression was associated with a better OS in resected NSCLC mainly squamous cell histology, with larger tumour size, positive lymph node status and those receiving the adjuvant therapy (37). But recently, in a large cohort of resected NSCLC with nodal involvement from $\mathrm{N} 0$ to $\mathrm{N} 2$, no association was shown between PD-L1 expression (defined as more than $50 \%$ of tumour cells positivity) and survival except for patients with $\mathrm{N} 2$ disease. In this group, a strong PD-L1 expression was associated with significantly improved DFS and OS on a multivariate analysis (38).

To investigate the prognostic and predictive role of PD-L1 expression in early NSCLC patients receiving an adjuvant treatment, tumour sections from three pivotal adjuvant chemotherapy trials (IALT, JBR.10, CALGB 9633) (39-41) were tested for its expression on tumour and immune cells. Specimens from 982 patients were analysed and PD-L1 positivity was found to be associated with squamous histology, intense lymphocytic infiltrate and KRAS mutation, while EGFR mutated tumours showed a statistically non-significant lower expression. However, the final analysis showed that the PD-L1 expression was neither prognostic nor predictive for survival benefit from adjuvant chemotherapy (42).

Recently, Usò et al. investigated the prognostic role of several immune biomarkers in a cohort of resected NSCLC patients, evaluating the relative expression of 20 immunerelated genes. Patients presenting a higher expression of interleukine-23A and LGALS2 (Galectine-2) presented a better outcome as well as patients with higher expression of immunoregulatory genes as CTLA4 and interleukine- 10 . These results were used to develop a gene expression score and, since CTLA4 and PD-1 were found associated with prognosis, a prediction model including these two genes was created. High immune checkpoint score correlated with a longer OS and a longer relapse-free survival (RFS), being an independent prognostic factor (43).

At the same time, Paulsen et al. investigated the expression of PD-L1 and PD-1 in early NSCLC and their potential use in a prognostic immunoscore to include into the TNM staging classification. Tissue samples of 536 resected stage I to IIIA NSCLC were analysed for PD-L1 and PD-1 expression in the primary tumour and metastatic lymph node tissue. Multivariate analysis showed that PDL1 positive immune cells in the stromal compartment and $\mathrm{PD}-1$ positive tumour infiltrating lymphocytes were independent positive prognostic factors while, combined low scores, remained an independent prognostic factor for poor survival (44).

All these evidences suggest a potential role of host immune response and in particular of immune checkpoints in influencing survival of early NSCLC. However, no sufficient data are available to support the assessment of any parameters linked to the innate or adaptive immune response in the resected tissue as biomarker of outcomes prediction in early NSCLC. Therefore, no tissue characteristics can be currently used to identify patients that could achieve a greater benefit from adjuvant treatment. However, they provide a strong rational to speculate about a potential role of immunotherapy in this setting.

\section{Evidences of immunotherapy efficacy in early NSCLC}

Considering all these evidences, different strategies have been evaluated to improve the early NSCLC outcomes eliciting the anti-tumour immune response. Among these, tumour vaccines and cellular therapies were the first investigated (Table 1).

Tumour vaccines can induce an in vivo immune response producing antibodies, cytotoxic $\mathrm{T}$ cells and $\mathrm{T}$ helper cells against tumour-associated antigens formulated in the vaccine. The efficacy of vaccine against melanoma- 
Table 1 Randomized trials

\begin{tabular}{|c|c|c|c|c|c|}
\hline Study & Phase & Stage & Therapeutic intervention & Accrual & Primary endpoint \\
\hline Kimura 1997 (46) & III & I-IV & $\begin{array}{l}\text { Either adjuvant chemotherapy or radiotherapy +/- } \\
\qquad I L-2 / L A K\end{array}$ & 170 & OS \\
\hline Zhao 2014 (48) & NR & III & Adjuvant chemotherapy +/- DC-CIK & 157 & DFS, RR, OS \\
\hline Kimura 2015 (49) & III & Ib-IV & Adjuvant chemotherapy $+/-$ AKT-DC & 103 & OS \\
\hline Vansteenkiste 2016 (50) & III & lb-IIIa & $\begin{array}{l}\text { Standard adjuvant chemotherapy or not }+ \text { /- adjuvant } \\
\text { MAGE-A3 }\end{array}$ & 2,312 & DFS \\
\hline
\end{tabular}

IL-2, interleukin 2; rIL-2, recombinant interleukin 2; LAK, lymphokine-activated killer; AKT-DC, activated killer T cells and dendritic cells; DC-CIK, dendritic cells and cytokine-induced killer cells; TIL, tumor-infiltrating lymphocytes; MAGE-A3, vaccine against melanomaassociated antigen; OS, overall survival; DFS, disease-free survival; DFI, disease-free interval; RR, recurrence rate; NR, not reported.

associated antigen (MAGE-A3) has been evaluated in early NSCLC setting. The rationale to develop this vaccine was that this antigen is almost exclusively express on tumour cells and not on normal tissues (51), and its expression ranges from $25 \%$ to $50 \%$ in non-squamous and squamous cells NSCLC respectively (52).

On 2013 the results of the phase II trial evaluating the clinical activity, the immunological response and safety of MAGE-A3 vaccine in completely resected MAGE-A3 positive stage IB-II NSCLC were published (47). After the surgical intervention, 183 MAGE-A3 positive patients were directly randomized to either vaccine or placebo. No statistically significant improvement in disease-free interval (DFI) [hazard ratio (HR) $=0.75 ; 95 \%$ CI, 0.46-1.23; $\mathrm{P}=0.254)$, DFS (HR $=0.76 ; 95 \% \mathrm{CI}, 0.48-1.21 ; \mathrm{P}=0.248)]$ or OS (HR $=0.81 ; 95 \%$ CI, 0.47-1.40; $\mathrm{P}=0.454$ ) was observed. The main weaknesses of the study were the limited sample size and the absence of adjuvant chemotherapy.

The MAGRIT trial, a large double blind, placebo controlled, phase III trial enrolling 2,312 completely resected stage IB to IIIA MAGE-A3 positive NSCLC who did or did not receive adjuvant chemotherapy, tried to overcome these limitations (50). No statistically significant difference was detected in term of DFS in the overall population (HR $=1.02 ; 95 \% \mathrm{CI}, 0.89-1.18 ; \mathrm{P}=0.74$ ) or in patients that did not receive an adjuvant chemotherapy ( $\mathrm{HR}=0.97 ; 95 \% \mathrm{CI}, 0.80-1.18 ; \mathrm{P}=0.76)$. Considering the disappointing results reported further developments of MAGE-A3 vaccine in early NSCLC setting has been stopped.
Another strategy evaluated in the past few years was the adoptive cell transfer. This approach consists in the isolation of host antigen-specific cells, their ex vivo expansion and activation, and subsequent autologous administration to enhance the tumour-specific immune response. Four randomized trial evaluated the efficacy of different autologous adoptive cellular therapies (interleukine- 2 combined with tumour-infiltrating lymphocytes or lymphokine-activated killer cells, activated killer T cells associated to DCs, and DCs associated to cytokine-induced killer cells) $(45,46,48,49)$ in early NSCLC and all of them were conducted in adjuvant setting. All these trials showed a better outcome in patients treated in the experimental arms receiving the adoptive cells therapy compared to the control ones.

Then, to better understands the efficacy of this strategy in adjuvant setting, a meta-analysis tried to resume these results comparing the experimental arms of these randomized trials (472 patients) to the control therapies. The adoptive immunotherapy showed a significant benefit on survival ( $\mathrm{HR}=0.61 ; 95 \% \mathrm{CI}, 0.45-0.84 ; \mathrm{P}=0.002$ ) and a $39 \%$ reduction in the relative risk of death compared to control arm without differences between trials (53).

Despite these positive results, the studies have not sufficient statistical power to provide clear information about the efficacy of adoptive cellular therapies in adjuvant setting. Indeed, the sample sizes were modest, the variability of population included made results not comparable, they often included also locally advanced and stage IV diseases, and some of them were conducted before the introduction of adjuvant chemotherapy so, underpowered 
treatment strategies were used as control arm in some cases. Furthermore, adoptive cellular therapies present major limitations due to their costs and the specialized cellproduction facilities along with a highly trained laboratory and medical staff required to their production.

Regarding all these limitations, adoptive cellular therapy has recently become less attractive in early NSCLC setting but several studies are still investigating their potential role in advanced patients.

\section{Future directions: immune checkpoints inhibitors}

Considering the modest results obtained by vaccine, the difficulties in the development of adoptive cellular therapies and following the impressive results of the immune checkpoint inhibitors in the locally advanced and metastatic settings, most of clinical trials currently ongoing are exploring the efficacy of immunotherapy in the neoadjuvant and adjuvant fields (Table 2).

Some data about the potential role of immune checkpoint inhibitors as neo-adjuvant treatment are already available. A phase II study enrolled 24 stage I to IIIA NSCLC patients to receive neo-adjuvant therapy (three cycles of paclitaxel with either cisplatin or carboplatin) and ipilimumab from the second cycle. This study did not meet the primary endpoint of an increasing tumour-associatedantigens $T$ cell response after ipilimumab administration but, it highlighted the immune activating properties of ipilimumab in early NSCLC. In fact, a weighty activation of both CD4+ and CD8+ cells were observed after ipilimumab administration, with a significant frequency increase of activated CD4+ cells expressing markers as ICOS (Inducible T-cell Costimulator), HLA-DR, CTLA4 and PD-1, and of activated CD8+ cells expressing all the previous markers with the exception of PD-1. On the other hand ipilimumab showed a little effect on circulating Treg and MDSC (54).

Recently, preliminary results from another phase II clinical trial conducted on 22 stage I to IIIA NSCLC patients treated with neo-adjuvant nivolumab were presented (55). The primary endpoint was safety and it was met thanks to a well-tolerated treatment without any surgery delay required. Efficacy was explored using the objective pathological response criteria and $43 \%$ of resected tumour (95\% CI, 24-63\%) had a major pathological response, defined as less than $10 \%$ of viable tumour cells in the resected specimens. Correlative studies about tumour microenvironment showed an association between both pretreatment tumour mutations and neoantigens loads with pathologic response, while immunologic analyses confirmed the presence of intratumoral $\mathrm{T}$ cell clones in the blood after nivolumab treatment (55).

These results open the door to a wide range of investigations to better understand the real role of immune checkpoint inhibitors in neo-adjuvant and adjuvant settings in term of pathological response, tumour microenvironment and peripheral compartment modulation and clinical benefit (Table 2).

\section{Immune checkpoints inbibitors and chemotherapy combinations}

The combination of immunotherapy with chemotherapy may engage an additive or synergistic clinical activity. Chemotherapy promotes tumour immunity through the induction of immunogenic cell death and disruption of the strategies used by the tumour to avoid the immune recognition. Chemotherapy agents may enhance the tumour immunogenicity in different ways, as by up-regulating the tumour antigen expression or the MHC Class I molecules, up-regulating or down-regulating co-stimulatory or coinhibitory molecules respectively on the tumour cell surface, or making tumour cells more sensitive to $\mathrm{T}$ cell-mediated lysis. All these mechanism cause an enhancement of the effector T cell activity $(56,57)$.

The optimal timing for the integration of immunotherapy with chemotherapy to minimize side effects and engage the potential synergy is unknown, but an obvious strategy could be using immunotherapy in the setting of minimal residual disease after the surgical resection and adjuvant chemotherapy. For that reason, early NSCLC seems to be a really appealing setting to investigate the efficacy of immunotherapy in combination with standard chemotherapy and many trials are currently investigating the synergistic effect of a checkpoint inhibitor combined to the standard neo-adjuvant and/or adjuvant chemotherapy (Table 2).

\section{Immune checkpoint inhibitors and radiotherapy combinations}

Some evidences in different tumor subtypes show that the combination of immunotherapy and radiotherapy may produce a synergistic effect (58). Radiation can cause the release of tumor antigens via inflammatory cell death, the activation and migration of DCs and cross-presentation of tumor antigens resulting in tumor-specific T-cell activation and proliferation (59-61). Radiotherapy may also 
Table 2 Ongoing trials

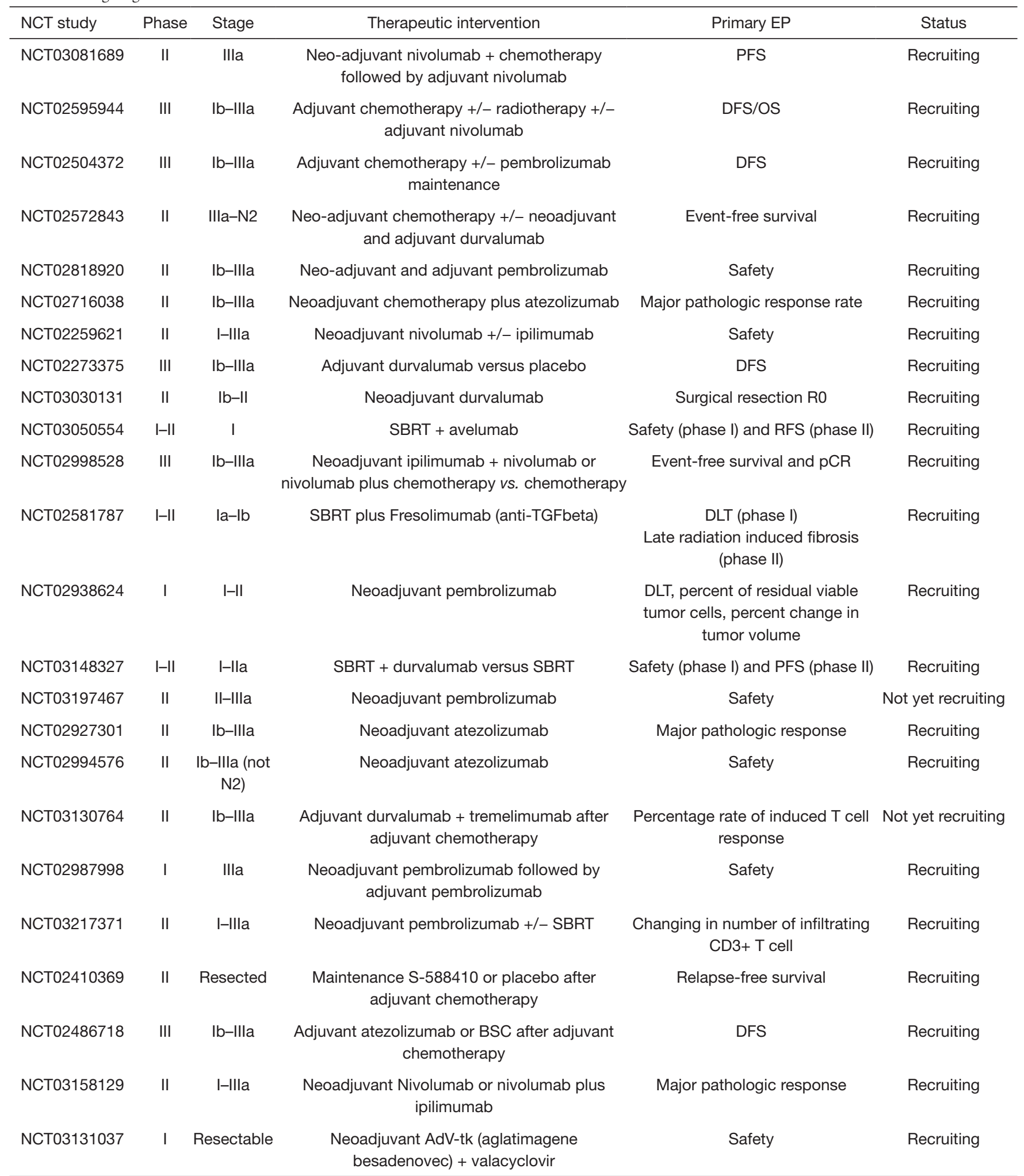

Clinicaltrial.gov research updated to December $6{ }^{\text {th }}$ 2017. EP, endpoint; OS, overall survival; DFS, disease-free survival; DFI, disease-free interval; PFS, progression-free survival; RFS, relapse-free survival; pCR, pathological complete response; DLT, dose-limiting toxicities. 
modulate the expression of immune-checkpoint ligands, including PD-L1, on tumor cells surface and on the tumor microenvironment immune cells. Then, a subsequent use of an anti-PD-L1 agent might enhance the therapeutic efficacy of ionizing radiotherapy (62). Furthermore, in some patient reports, radiotherapy may induce an abscopal effect improving the distance disease control (63). Consequently, the administration of immune checkpoint inhibitors during or after a radiotherapy treatment could improve the local and distance RFS by reducing the immunesuppressive microenvironment. Currently, the real benefit of radiotherapy combined with immunotherapy in early NSCLC treatment remain indeterminate together with the safety of this combination, mainly in term of pulmonary toxicities $(5,6)$. Grade 3 or higher pneumonitis may be seen in around $5 \%$ of patients after treatment with checkpoint inhibitors as single agent or in combined schedule and it is unknown the magnitude of synergy between radiotherapy and checkpoint modulators (64). The potential role of SBRT to enhance the immunotherapy efficacy justifies the ongoing trials dedicated to very early NSCLC disease (Table 2).

\section{Immune checkpoint inbibitors combinations}

The immunoinhibition of $\mathrm{T}$ cells by the use of checkpoint inhibitors may open the door for combinations with a large number of immunotherapies able to improve the proportion of responding patients. Several clinical trials are currently exploring combinations with agents that may directly stimulate cytotoxic cells, block tumour-expressed immunoinhibitory factors, inhibit regulatory $\mathrm{T}$ cells, block the inhibition of natural killer cells or block the activity of soluble factors produced by stromal myeloid and mesenchymal cells (65). Unfortunately, most of these studies are focused on advanced diseases but they could provide interesting information to transpose on early NSCLC in the future (Table 2).

\section{Duration of immune checkpoint inbibitors treatment}

One of the open questions about immune checkpoint inhibitors is the treatment duration. Patients longer responders after checkpoint inhibitors discontinuation in advanced setting are usually found in daily-clinical practice and treatment administration until progression may rise up some issue like cumulative side effects, inconvenience of coming to hospital for infusions every 2-3 weeks, and major expense. In spite the optimal duration of immune checkpoint inhibitors is not known yet, some data about early discontinuation are already available in melanoma and NSCLC. In advanced melanoma patients, a pooled analysis of the CheckMate 069 (nivolumab plus ipilimumab or ipilimumab alone in metastatic melanoma) and the CheckMate 067 (nivolumab or nivolumab plus ipilimumab $v s$. ipilimumab alone in advanced melanoma) trials comparing patients who discontinued because of adverse events during the induction phase with ipilimumab and those who did not, showed similar PFS (8.4 vs. 10.8 months, $\mathrm{P}=0.97)$ and objective response rate $(58.3 \%$ vs. $50.2 \%)$ in the two groups (66-68). Besides, a retrospective review on 29 melanoma patients achieved a complete response to anti PD-1 (pembrolizumab or nivolumab) therapy showed a median time off therapy after discontinuation within 4.5 and 10 months (69). Therefore, even after discontinuation, many advanced melanoma patients may continue to derive benefit from immune checkpoint inhibitors. It is less clear if the same results can be expected also in NSCLC patients. Recently, Spiegel et al. presented the preliminary results of CheckMate 153 trial, an ongoing phase IIIB/IV study evaluating the clinical benefit of a fixed-duration (1 year) of nivolumab treatment versus continuous treatment in patients with previously treated advanced NSCLC. Patients who were stable or responders after 1 year of nivolumab were randomized to either continue receiving treatment or to stop it. The primary objective was the incidence of high-grade selected treatment-related adverse events, while prespecified exploratory endpoints included safety and efficacy. On 218 patients enrolled at time of analysis, a slightly higher rate of treatment-related adverse events was observed in the continuous treatment arm (39\% vs. $25 \%)$, as well as grade $3-4$ toxicities ( $8 \%$ vs. $4 \%$ ). Interesting, median PFS from randomization was 10.3 months in the 1-year treatment arm and was not reached in the continuous treatment one. The 6-month and 1-year PFS rate supported a higher benefit for the continuous treatment arm $(69 \%$ vs. $80 \%$ and $40 \%$ vs. $65 \%$, respectively), without differences in the hazard ratio for PFS between those who had a complete or partial response and those who showed a stable disease. A trend for OS improvement was also detected in the continuous treatment arm (median OS 23.2 months in the 1-year treatment arm and not reached in the continuous one) (70). Considering these contradictory data, the immune checkpoints treatment duration still represents an important issue to explore in randomized prospective trials. Then, most of clinical trials currently investigating the immune checkpoints inhibitors in the 
early NSCLC setting provide an arbitrary limited treatment course of 12-24 months.

\section{Conclusions}

Targeting the immune system to elicit the host immunological response against cancer could be an effective way to reduce relapse and improve survival of early NSCLC.

In the past few years, two strategies have been investigated with this objective (MAGE-A3 vaccine and the adoptive cell transfer), but none of them provided convincing results. If the former did not show any clinical benefit in trials conducted on early NSCLC, the latter provided a positive trend in all studies performed, but the lack of more consistent data deriving from larger cohorts with updated comparators and the limitations in daily-life applicability made this strategy less attractive. Preclinical and clinical data supported the prognostic role of immune checkpoints in resected NSCLC even if they did not show a clear predictive value for adjuvant treatment benefit. However, some preliminary data about the safety and the efficacy of neo-adjuvant immune checkpoint inhibitors encourage to further investigate their potential role in early settings as monotherapy or as part of a multimodal strategy. Then, even if no significant advances have been reached in early NSCLC treatment until today, checkpoint inhibitors can open the door to a new strategy in this field in the near future.

\section{Acknowledgements}

None.

\section{Footnote}

Conflicts of Interest: The authors have no conflicts of interest to declare.

\section{References}

1. Goldstraw P, Chansky K, Crowley J, et al. The IASLC Lung Cancer Staging Project: Proposals for Revision of the TNM Stage Groupings in the Forthcoming (Eighth) Edition of the TNM Classification for Lung Cancer. J Thorac Oncol 2016;11:39-51.

2. Howlader N, Noone AM, Krapcho M, et al. SEER Cancer Statistics Review, 1975-2014, National Cancer Institute. Bethesda, MD, https://seer.cancer.gov/csr/1975_2014/, based on November 2016 SEER data submission, posted to the SEER web site, April 2017.

3. Artal Cortés Á, Calera Urquizu L, Hernando Cubero J. Adjuvant chemotherapy in non-small cell lung cancer: state-of-the-art. Transl Lung Cancer Res 2015;4:191-7.

4. Rosen JE, Keshava HB, Yao X, et al. The Natural History of Operable Non-Small Cell Lung Cancer in the National Cancer Database. Ann Thorac Surg 2016;101:1850-5.

5. Lindberg K, Nyman J, Riesenfeld Källskog V, et al. Longterm results of a prospective phase II trial of medically inoperable stage I NSCLC treated with SBRT - the Nordic experience. Acta Oncol 2015;54:1096-104.

6. Verstegen NE, Lagerwaard FJ, Hashemi SM, et al. Patterns of Disease Recurrence after SABR for Early Stage Non-Small-Cell Lung Cancer: Optimizing FollowUp Schedules for Salvage Therapy. J Thorac Oncol 2015;10:1195-200.

7. Lou F, Huang J, Sima CS, et al. Patterns of recurrence and second primary lung cancer in early-stage lung cancer survivors followed with routine computed tomography surveillance. J Thorac Cardiovasc Surg 2013;145:75-81; discussion 81-2.

8. Reck M, Rodríguez-Abreu D, Robinson AG, et al. Pembrolizumab versus Chemotherapy for PD-L1Positive Non-Small-Cell Lung Cancer. N Engl J Med 2016;375:1823-33.

9. Brahmer J, Reckamp KL, Baas P, et al. Nivolumab versus Docetaxel in Advanced Squamous-Cell Non-Small-Cell Lung Cancer. N Engl J Med 2015;373:123-35.

10. Borghaei H, Paz-Ares L, Horn L, et al. Nivolumab versus Docetaxel in Advanced Nonsquamous Non-Small-Cell Lung Cancer. N Engl J Med 2015;373:1627-39.

11. Antonia SJ, Villegas A, Daniel D, et al. Durvalumab after Chemoradiotherapy in Stage III Non-Small-Cell Lung Cancer. N Engl J Med 2017;377:1919-29.

12. Johnson SK, Kerr KM, Chapman AD, et al. Immune cell infiltrates and prognosis in primary carcinoma of the lung. Lung Cancer 2000;27:27-35.

13. Al-Shibli KI, Donnem T, Al-Saad S, et al. Prognostic effect of epithelial and stromal lymphocyte infiltration in nonsmall cell lung cancer. Clin Cancer Res 2008;14:5220-7.

14. Dai F, Liu L, Che G, et al. The number and microlocalization of tumor-associated immune cells are associated with patient's survival time in non-small cell lung cancer. BMC Cancer 2010;10:220.

15. Kawai O, Ishii G, Kubota K, et al. Predominant infiltration of macrophages and CD8(+) T Cells in cancer nests is a significant predictor of survival in stage IV nonsmall cell lung cancer. Cancer 2008;113:1387-95. 
16. Talmadge JE, Donkor M, Scholar E. Inflammatory cell infiltration of tumors: Jekyll or Hyde. Cancer Metastasis Rev 2007;26:373-400.

17. Bremnes RM, Al-Shibli K, Donnem T, et al. The role of tumor-infiltrating immune cells and chronic inflammation at the tumor site on cancer development, progression, and prognosis: emphasis on non-small cell lung cancer. J Thorac Oncol 2011;6:824-33.

18. Welsh TJ, Green RH, Richardson D, et al. Macrophage and mast-cell invasion of tumor cell islets confers a marked survival advantage in non-small-cell lung cancer. J Clin Oncol 2005;23:8959-67.

19. Al-Shibli K, Al-Saad S, Donnem T, et al. The prognostic value of intraepithelial and stromal innate immune system cells in non-small cell lung carcinoma. Histopathology 2009;55:301-12.

20. Ma J, Liu L, Che G, et al. The M1 form of tumorassociated macrophages in non-small cell lung cancer is positively associated with survival time. BMC Cancer 2010;10:112.

21. Ohri CM, Shikotra A, Green RH, et al. Macrophages within NSCLC tumour islets are predominantly of a cytotoxic M1 phenotype associated with extended survival. Eur Respir J 2009;33:118-26.

22. Inoshima N, Nakanishi Y, Minami T, et al. The influence of dendritic cell infiltration and vascular endothelial growth factor expression on the prognosis of non-small cell lung cancer. Clin Cancer Res 2002;8:3480-6.

23. Gottlin EB, Bentley RC, Campa MJ, et al. The Association of Intratumoral Germinal Centers with early-stage nonsmall cell lung cancer. J Thorac Oncol 2011;6:1687-90.

24. Dieu-Nosjean MC, Antoine M, Danel C, et al. Longterm survival for patients with non-small-cell lung cancer with intratumoral lymphoid structures. J Clin Oncol 2008;26:4410-7.

25. Joyce JA, Fearon DT. T cell exclusion, immune privilege, and the tumor microenvironment. Science 2015;348:74-80.

26. Carus A, Ladekarl M, Hager H, et al. Tumor-associated neutrophils and macrophages in non-small cell lung cancer: no immediate impact on patient outcome. Lung Cancer 2013;81:130-7.

27. Oleinika K, Nibbs RJ, Graham GJ, et al. Suppression, subversion and escape: the role of regulatory $\mathrm{T}$ cells in cancer progression. Clin Exp Immunol 2013;171:36-45.

28. Mittal D, Gubin MM, Schreiber RD, et al. New insights into cancer immunoediting and its three component phases--elimination, equilibrium and escape. Curr Opin Immunol 2014;27:16-25.
29. Salvi S, Fontana V, Boccardo S, et al. Evaluation of CTLA4 expression and relevance as a novel prognostic factor in patients with non-small cell lung cancer. Cancer Immunol Immunother CII 2012;61:1463-72.

30. Deng L, Gyorffy B, Na F, et al. Association of PDCD1 and CTLA-4 Gene Expression with Clinicopathological Factors and Survival in Non-Small-Cell Lung Cancer: Results from a Large and Pooled Microarray Database. J Thorac Oncol 2015;10:1020-6.

31. Walunas TL, Lenschow DJ, Bakker CY, et al. CTLA-4 can function as a negative regulator of $\mathrm{T}$ cell activation. Immunity 1994;1:405-13.

32. Linsley PS, Greene JL, Tan P, et al. Coexpression and functional cooperation of CTLA-4 and CD28 on activated T lymphocytes. J Exp Med 1992;176:1595-604.

33. Jago CB, Yates J, Câmara NO, et al. Differential expression of CTLA-4 among T cell subsets. Clin Exp Immunol 2004;136:463-71.

34. Freeman GJ, Long AJ, Iwai Y, et al. Engagement of the PD-1 immunoinhibitory receptor by a novel B7 family member leads to negative regulation of lymphocyte activation. J Exp Med 2000;192:1027-34.

35. Creelan BC. Update on immune checkpoint inhibitors in lung cancer. Cancer Control 2014;21:80-9.

36. Velcheti V, Schalper KA, Carvajal DE, et al. Programmed death ligand-1 expression in non-small cell lung cancer. Lab Investig 2014;94:107-16.

37. Schmidt LH, Kümmel A, Görlich D, et al. PD-1 and PDL1 Expression in NSCLC Indicate a Favorable Prognosis in Defined Subgroups. PLoS One 2015;10:e0136023.

38. Ameratunga M, Asadi K, Lin X, et al. PD-L1 and Tumor Infiltrating Lymphocytes as Prognostic Markers in Resected NSCLC. PLoS One 2016;11:e0153954.

39. Arriagada R, Bergman B, Dunant A, et al. Cisplatinbased adjuvant chemotherapy in patients with completely resected non-small-cell lung cancer. N Engl J Med 2004;350:351-60.

40. Butts CA, Ding K, Seymour L, et al. Randomized phase III trial of vinorelbine plus cisplatin compared with observation in completely resected stage IB and II nonsmall-cell lung cancer: updated survival analysis of JBR-10. J Clin Oncol 2010;28:29-34.

41. Strauss GM, Herndon JE, Maddaus MA, et al. Adjuvant paclitaxel plus carboplatin compared with observation in stage IB non-small-cell lung cancer: CALGB 9633 with the Cancer and Leukemia Group B, Radiation Therapy Oncology Group, and North Central Cancer Treatment Group Study Groups. J Clin Oncol 2008;26:5043-51. 
42. Tsao MS, Le Teuff G, Shepherd FA, et al. PD-L1 protein expression assessed by immunohistochemistry is neither prognostic nor predictive of benefit from adjuvant chemotherapy in resected non-small cell lung cancer. Ann Oncol 2017;28:882-9.

43. Usó M, Jantus-Lewintre E, Calabuig-Fariñas S, et al. Analysis of the prognostic role of an immune checkpoint score in resected non-small cell lung cancer patients. Oncoimmunology 2016;6:e1260214.

44. Paulsen EE, Kilvaer TK, Khanehkenari MR, et al. Assessing PDL-1 and PD-1 in Non-Small Cell Lung Cancer: A Novel Immunoscore Approach. Clin Lung Cancer 2017;18:220-33.e8.

45. Ratto GB, Zino P, Mirabelli S, et al. A randomized trial of adoptive immunotherapy with tumor-infiltrating lymphocytes and interleukin-2 versus standard therapy in the postoperative treatment of resected nonsmall cell lung carcinoma. Cancer 1996;78:244-51.

46. Kimura H, Yamaguchi Y. A phase III randomized study of interleukin-2 lymphokine-activated killer cell immunotherapy combined with chemotherapy or radiotherapy after curative or noncurative resection of primary lung carcinoma. Cancer 1997;80:42-9.

47. Vansteenkiste J, Zielinski M, Linder A, et al. Adjuvant MAGE-A3 immunotherapy in resected non-small-cell lung cancer: phase II randomized study results. J Clin Oncol 2013;31:2396-403.

48. Zhao M, Li H, Li L, et al. Effects of a gemcitabine plus platinum regimen combined with a dendritic cell-cytokine induced killer immunotherapy on recurrence and survival rate of non-small cell lung cancer patients. Exp Ther Med 2014;7:1403-7.

49. Kimura H, Matsui Y, Ishikawa A, et al. Randomized controlled phase III trial of adjuvant chemoimmunotherapy with activated killer $\mathrm{T}$ cells and dendritic cells in patients with resected primary lung cancer. Cancer Immunol Immunother 2015;64:51-9.

50. Vansteenkiste JF, Cho BC, Vanakesa T, et al. Efficacy of the MAGE-A3 cancer immunotherapeutic as adjuvant therapy in patients with resected MAGE-A3-positive nonsmall-cell lung cancer (MAGRIT): a randomised, doubleblind, placebo-controlled, phase 3 trial. Lancet Oncol 2016;17:822-35.

51. De Plaen E, Arden K, Traversari C, et al. Structure, chromosomal localization, and expression of 12 genes of the MAGE family. Immunogenetics 1994;40:360-9.

52. Kim JH, Jassem J, Tada H, et al. MAGE-A3 gene expression frequency and demography data of stage IB-
IIIA NSCLC patients from ongoing MAGRIT Phase III trial evaluating MAGE-A3 Antigen Specific Cancer Immunotherapeutic (ASCI) as adjuvant treatment. ESMO 2010, Abstract 4935.

53. Zeng Y, Ruan W, He J, et al. Adoptive Immunotherapy in Postoperative Non-Small-Cell Lung Cancer: A Systematic Review and Meta-Analysis. PLoS One 2016;11:e0162630.

54. Yi JS, Ready N, Healy P, et al. Immune Activation in Early-Stage Non-Small Cell Lung Cancer Patients Receiving Neoadjuvant Chemotherapy Plus Ipilimumab. Clin Cancer Res 2017;23:7474-82.

55. Chaft JE, Forde PM, Smith KN, et al. Neoadjuvant nivolumab in early-stage, resectable non-small cell lung cancers. J Clin Oncol 2017;35:8508.

56. Chen G, Emens LA. Chemoimmunotherapy: reengineering tumor immunity. Cancer Immunol Immunother CII 2013;62:203-16.

57. Emens LA, Middleton G. The interplay of immunotherapy and chemotherapy: harnessing potential synergies. Cancer Immunol Res 2015;3:436-43.

58. Sharabi AB, Lim M, DeWeese TL, et al. Radiation and checkpoint blockade immunotherapy: radiosensitisation and potential mechanisms of synergy. Lancet Oncol 2015;16:e498-509.

59. Burnette BC, Liang H, Lee Y, et al. The efficacy of radiotherapy relies upon induction of type $\mathrm{i}$ interferondependent innate and adaptive immunity. Cancer Res 2011;71:2488-96.

60. Gupta A, Probst HC, Vuong V, et al. Radiotherapy promotes tumor-specific effector CD8+ T cells via dendritic cell activation. J Immunol 2012;189:558-66.

61. Parker JJ, Jones JC, Strober S, et al. Characterization of direct radiation-induced immune function and molecular signaling changes in an antigen presenting cell line. Clin Immunol 2013;148:44-55.

62. Deng L, Liang H, Burnette B, et al. Irradiation and antiPD-L1 treatment synergistically promote antitumor immunity in mice. J Clin Invest 2014;124:687-95.

63. Siva S, MacManus MP, Martin RF, et al. Abscopal effects of radiation therapy: a clinical review for the radiobiologist. Cancer Lett 2015;356:82-90.

64. Boutros C, Tarhini A, Routier E, et al. Safety profiles of anti-CTLA-4 and anti-PD-1 antibodies alone and in combination. Nat Rev Clin Oncol 2016;13:473-86.

65. Mahoney KM, Rennert PD, Freeman GJ. Combination cancer immunotherapy and new immunomodulatory targets. Nat Rev Drug Discov 2015;14:561-84.

66. Hodi FS, Chesney J, Pavlick AC, et al. Combined 
nivolumab and ipilimumab versus ipilimumab alone in patients with advanced melanoma: 2-year overall survival outcomes in a multicentre, randomised, controlled, phase 2 trial. Lancet Oncol 2016;17:1558-68.

67. Wolchok JD, Chiarion-Sileni V, Gonzalez R, et al. Overall Survival with Combined Nivolumab and Ipilimumab in Advanced Melanoma. N Engl J Med 2017;377:1345-56.

68. Schadendorf D, Wolchok JD, Hodi FS, et al. Efficacy and Safety Outcomes in Patients With Advanced Melanoma Who Discontinued Treatment With Nivolumab and Ipilimumab Because of Adverse Events: A Pooled Analysis of Randomized Phase II and III Trials. J Clin Oncol 2017;35:3807-14.

69. Ladwa R, Atkinson V. The cessation of anti-PD-1 antibodies of complete responders in metastatic melanoma. Melanoma Res 2017;27:168-70.

70. Spigel DR, McLeod M, Hussein MA, et al. 1297ORandomized results of fixed-duration (1-yr) vs continuous nivolumab in patients (pts) with advanced nonsmall cell lung cancer (NSCLC). Ann Oncol 2017;28. Available online: https://academic.oup.com/annonc/ article/28/suppl_5/mdx380.002/4109357
Cite this article as: Gobbini E, Giaj Levra M. Is there a room for immune checkpoint inhibitors in early stage non-small cell lung cancer? J Thorac Dis 2018;10(Suppl 13):S1427-S1437. doi: $10.21037 /$ jtd.2018.01.81 